\title{
Percepção de profissionais do atendimento pré-hospitalar na assistência à urgência e emergência (crise) em psiquiatria
}

\author{
Perception of pre-hospital care professionals in urgency and emergency care (crisis) in psychiatry \\ Percepción de profesionales de atención prehospitalaria en el manejo de urgencia y emergencia \\ (crisis) en psiquiatría
}

Recebido: 13/08/2021 | Revisado: 19/08/2021 | Aceito: 29/08/2021 | Publicado: 31/08/2021

Tatiane dos Santos Souza Ramos

ORCID: https://orcid.org/0000-0001-8777-7979 Hospital das Clínicas Doutor Serafim de Carvalho, Brasil E-mail: enf_tatiramos@outlook.com

Matheus Almeida Ramalho

ORCID: https://orcid.org/0000-0002-5607-1313 Universidade Federal de Jataí, Brasil

E-mail: matheusramalho@discente.ufg.br

Tatiele dos Santos Souza

ORCID: https://orcid.org/0000-0003-1271-7263 Universidade Federal de Jataí, Brasil E-mail: enf_tatiramos@outlook.com

Cynthia Moraes Medeiros

ORCID: https://orcid.org/0000-0002-4615-9602 Universidade Federal de Jataí, Brasil

E-mail: moraes.cynhtia@gmail.com

Hellen Cristina Sthal

ORCID: https://orcid.org/0000-0001-8139-992X Universidade Federal de Jataí, Brasil

E-mail: hcsthal@ufg.br

Giulena Rosa Leite

ORCID: https://orcid.org/0000-0002-9294-0985 Universidade Federal de Jataí, Brasil

E-mail: giulenar@ufg.br

\begin{abstract}
Resumo
Objetivo: Identificar as evidências disponíveis na literatura nacional e internacional sobre as percepções de profissionais do Atendimento Pré-hospitalar (APH), em relação ao atendimento de urgência e emergência (crise) em psiquiatria. Método: Revisão integrativa de artigos publicados em inglês, português e espanhol. Para o levantamento, utilizou-se as bases de dados eletrônicas, BDENF, Lilacs e MEDLINE, utilizando os descritores de forma combinada "Atendimento pré-hospitalar" and "psiquiatria". A pesquisa foi realizada por meio da Rede BVS Brasileira/Biblioteca virtual em saúde em setembro de 2020, sendo selecionados sete artigos de forma criteriosa. Resultado: Na maioria dos estudos analisados, os resultados apontam que os profissionais que atuam no Serviço de Atendimento Móvel de Urgência (SAMU) apresentam receio, medo e falta de preparo no atendimento frente à crise psíquica, tornando o atendimento patologizante e estigmatizante. Conclusão: os profissionais do Serviço de Atendimento Móvel de Urgência (SAMU) ainda estão atrelados a um atendimento hospitalocêntrico, biomédico, de modelo manicomial, predominando o medo e uma lacuna no conhecimento técnico e científico. Apesar das mudanças adquiridas a partir da reforma psiquiátrica, o atendimento continua embasado na doença e no estigma que esta sugere.
\end{abstract}

Palavras-chave: Atendimento pré-hospitalar; Psiquiatria; Crise em saúde mental.

\begin{abstract}
Objective: To verify the evidence in the national and international literature on the perceptions of professionals in prehospital care, in relation to urgent and emergency care (crisis) in psychiatry. Method: Integrative review of articles published in English, Portuguese and Spanish. For the survey, electronic databases, BDENF, Lilacs and MEDLINE were used, using the descriptors in a combined way "Pre-hospital care" and "psychiatry". The research was carried out through the Brazilian VHL Network / Virtual Health Library in September 2020, with seven articles being carefully selected. Result: In most of the studies analyzed, the results indicate that professionals working in the Mobile Emergency Care Service (SAMU) have, fear, fear and lack of preparation in care in the face of a psychic crisis, making care pathological and stigmatizing. Conclusion: the professionals of the Mobile Emergency Care Service (SAMU) are still tied to hospital-centric, biomedical care with an asylum model, with fear predominating and
\end{abstract}


a gap in technical and scientific knowledge. Despite the changes acquired from the psychiatric reform, care remains based on the disease and the stigma it suggests.

Keywords: Prehospital care; Psychiatry; Crisis in mental health.

\begin{abstract}
Resumen
Objetivo: Identificar las evidencias disponibles en la literatura nacional e internacional referente a las percepciones de los profesionales de Atención Prehospitalaria (APS) en lo que respecta a la atención de urgencia y emergencia (crisis) en psiquiatría. Método: Revisión integradora de artículos publicados en inglés, portugués y español. Para la recolección de datos se utilizaron las bases de datos electrónicas BDENF, Lilacs y MEDLINE, se aplicaron los descriptores de manera combinada "Atención prehospitalaria" y "psiquiatría". La investigación se llevó a cabo a través de la Red BVS brasileña / Biblioteca Virtual en Salud en septiembre de 2020, con siete artículos seleccionados cuidadosamente. Resultado: En la mayoría de los estudios analizados, los resultados muestran que los profesionales que trabajan en el Servicio Móvil de Atención de Emergencias (SAMU) presentan temor, miedo y escasez de preparación en la entrega de atención ante una crisis relacionada con la salud mental, ya que esta se centra en la patología y el estigma. Conclusión: los profesionales del Servicio Móvil de Atención de Emergencias (SAMU) siguen vinculados a un modelo de atención hospitalocéntrico, biomédico, de modelo manicomial, con predominio del miedo, además de la brecha en el conocimiento técnico y científico. Pese a los cambios adquiridos tras la reforma psiquiátrica, la atención sigue basándose en la enfermedad y el estigma que esta acarrea.
\end{abstract}

Palabras clave: Atención prehospitalaria; Psiquiatría; Crisis de salud mental.

\title{
1. Introdução
}

A Reforma psiquiátrica no Brasil teve início em meados dos anos 70, mesma época da eclosão do "movimento sanitário", com o objetivo de desconstruir a lógica de exclusão manicomial e com intuito da humanização, equidade na oferta dos serviços, em defesa da saúde coletiva, desmistificando o conceito de periculosidade do louco, trazendo a garantia dos direitos com autonomia, e liberdade de cidadania.

Trata-se de um processo social complexo, que envolve a mudança na assistência à saúde, de acordo com os novos pressupostos técnicos e éticos sociais, abrangendo várias esferas políticas e instituições de forças diferentes, tornando-se um movimento sociopolítico que ocorre no âmbito da saúde pública, buscando garantias de cidadania para o indivíduo em sofrimento psíquico, compreendida como um conjunto de transformações de práticas, saberes, valores culturais e sociais. Assim, a reforma psiquiátrica avança com conflitos e desafios (Brasil, 2005).

A partir da promulgação da lei 10.216, de 2001, foram criados critérios mais rígidos para o funcionamento de hospitais psiquiátricos, diminuindo assim as internações e os leitos disponíveis. Em contrapartida, foram criados serviços com atenção integral, visando aos cuidados com uma abordagem terapêutica, trabalhando serviços psicoeducacionais e a reinserção social dos pacientes (Bonfada et. al., 2010).

Com as mudanças geradas pela reforma, surge o programa "De volta para casa" (PVC), criado pela Lei $n^{\circ} 10.708$, de 31 de julho de 2003, com o objetivo de reintegração social, visando o direito do paciente psiquiátrico de morar e conviver em liberdade. O PVC não é o único programa promovido pelo Ministério da Saúde desde a Reforma Psiquiátrica, nesse período, o Brasil também instituiu a Rede de Atenção Psicossocial (RAPS), articulando diferentes serviços, os Centros de Atenção Psicossocial (CAPS), leitos de saúde mental em Hospitais Gerais, Serviços Residenciais Terapêuticos (SRTs), Unidades de Acolhimento (UA), atendimento em serviços de atenção básica, e a articulação com os serviços de urgência e emergência, o qual é realizado e promovido pelo Serviço Móvel de atendimento às Urgências - SAMU, que se regulamenta pela portaria 2.048/GM e identifica a crise em saúde mental como urgência psiquiátrica, atribuindo a responsabilidade do atendimento ao SAMU (Brasil, 2002).

No entanto, essa responsabilidade atribuída a estes profissionais não pode ser transferida de forma incerta, sem uma adequada preparação para compreensão da complexidade da ação e da concretude de uma crise psíquica. A urgência em saúde mental é definida como quando o sujeito em crise necessita de um atendimento rápido, buscando uma resolução ou amenização do seu caos interno, e que este atendimento consiga evitar danos à integridade física e psíquica do indivíduo e de outros que 
estejam próximos a ele (Bonfada et. al., 2012).

O acolhimento implica numa relação humanizada com postura ética, é um vínculo entre o usuário e o serviço de saúde que resulta em uma escuta qualificada com resolução de suas queixas. Acolher é um compromisso de resposta às necessidades dos cidadãos que procuram os serviços de saúde (Brasil, 2015). O acolhimento entra como um papel fundamental no atendimento em crises psíquicas.

Assim, observa-se uma crescente demanda pelo atendimento na área da saúde mental em urgência e emergência, e observa-se uma dificuldade nesse atendimento, seja por falta de experiência, de treinamento em saúde mental, por não saberem como abordar o paciente ou até mesmo por medo de que haja alguma agressão física.

Este estudo contribui para as discussões nessa temática, visando à consolidação de uma rede de atenção à saúde mental que se aproxime das necessidades dos sujeitos em sofrimento psíquico, desconstruindo estereótipos, dissolvendo estigmas e promovendo cidadania e justiça social. Pesquisas que se ocupam dessas questões justificam a contribuição social da ciência, pois se engajam na busca por qualidade de vida, percebida enquanto emblema histórico dos profissionais e serviços de atenção à saúde mental.

O objetivo foi identificar as evidências disponíveis na literatura nacional e internacional sobre as percepções de enfermeiros, técnicos de enfermagem e médicos do Atendimento Pré-hospitalar (APH), em relação ao atendimento de urgência e emergência (crise) em psiquiatria.

\section{Metodologia}

Trata-se de um estudo de revisão integrativa da literatura cuja questão norteadora foi: quais as evidências na literatura nacional e internacional em relação ao atendimento pré-hospitalar sobre os atendimentos de urgências e emergências (crise) em psiquiatria?

Para o levantamento dos artigos, utilizou-se as bases de dados eletrônicas, BDENF, Lilacs e MEDLINE, utilizando os descritores de forma combinada "Atendimento pré-hospitalar" and "psiquiatria". A pesquisa foi realizada por meio da Rede BVS Brasileira/Biblioteca virtual em saúde em setembro de 2020.

Os critérios de inclusão definidos para a seleção dos artigos foram: artigos publicados em português, inglês e espanhol, com textos completos disponíveis online gratuitamente nas bases de dados eletrônicas, publicados e indexados nos referidos bancos de dados nos últimos dez anos.

Os critérios de exclusão foram: teses, artigos sem resumos, cartas, anais de congressos ou conferências, capítulos de livros, relatórios técnicos, editoriais, dissertações, artigos sem metodologia discriminada entre outros textos não científicos.

A amostra deu-se a partir da leitura dos títulos e resumos para verificar se os mesmos correspondiam à pergunta norteadora. Partindo-se desta análise foram selecionados todos os artigos que atenderam aos critérios de inclusão.

\section{Resultados}

Na primeira busca foram encontrados 581 artigos nas três bases de dados. Desses, 482 foram excluídos após aplicar os filtros com os critérios de elegibilidade, 99 artigos tiveram os títulos analisados, 84 foram excluídos pois os títulos não respondiam à pergunta norteadora, 15 artigos foram selecionados pelo título, 9 foram selecionados por resumos, 3 artigos repetidos sendo 2 excluídos, e 7 artigos lidos na íntegra e aceitos para o trabalho, conforme representado na Figura 1. 
Figura 1 - Etapas do processo de seleção dos artigos.

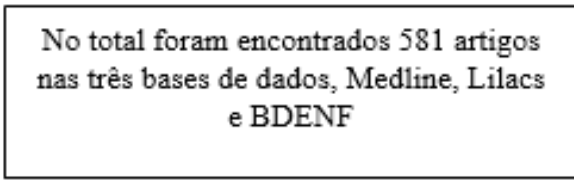

84 foram excluídos, pois não respondiam a questão norteadora.

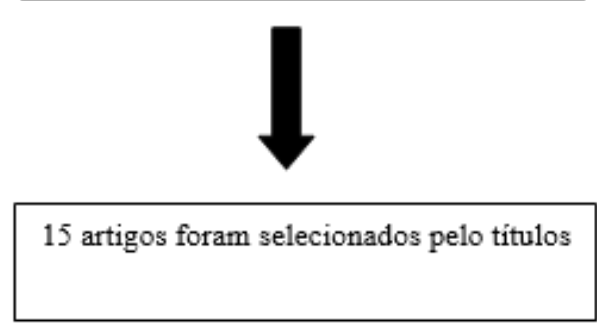

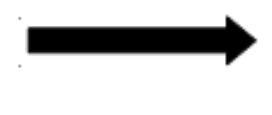
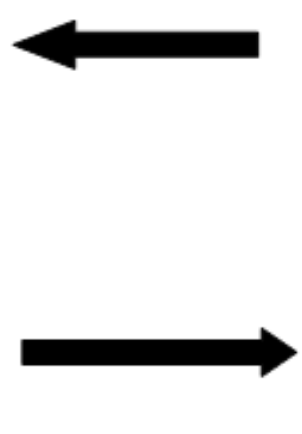

482 foram excluídos pelos critérios de elegibilidade

99 artigos tiveram os títulos analisados.

9 artigos foram escolhidos pelo resumo, houve 2 duplicações

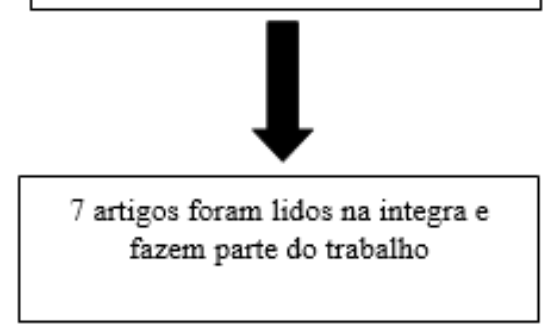

Fonte: Autores (2020).

Para a organização dos artigos utilizou-se um formulário, caracterizando-os segundo título, autores, fonte de publicação e ano, objetivo e nível de evidência.

A classificação dos níveis de evidências seguiu as recomendações Stillwell et. al. (2010), que consideram os níveis de evidência conforme o Quadro 1. 
Quadro 1 - Produções científicas: níveis de evidência.

\begin{tabular}{|c|c|c|}
\hline Tipo de evidência & Nível de evidência & Descrição do tipo de estudo \\
\hline $\begin{array}{lll}\text { Revisão } & \text { sistemática } & \text { ou } \\
\text { metanálise } & & \end{array}$ & NI & $\begin{array}{l}\text { Evidência proveniente de uma revisão sistemática ou } \\
\text { metanálise de ensaios clínicos randomizados controlados } \\
\text { ou oriundos de diretrizes baseadas em revisões } \\
\text { sistemáticas de ensaios clínicos a controlados }\end{array}$ \\
\hline Estudo randomizado controlado & NII & $\begin{array}{l}\text { Evidência obtida de pelo menos um ensaio clínico com } \\
\text { aleatorização, controlado e bem delineado / estudo } \\
\text { experimental }\end{array}$ \\
\hline $\begin{array}{l}\text { Estudo controlado com } \\
\text { randomização }\end{array}$ & NIII & $\begin{array}{l}\text { Evidência proveniente de um estudo bem desenhado e } \\
\text { controlado, sem aleatorização / quase experimental. }\end{array}$ \\
\hline $\begin{array}{l}\text { Estudo caso-controle ou estudo } \\
\text { de coorte }\end{array}$ & NIV & $\begin{array}{l}\text { Evidência proveniente de um estudo com desenho de } \\
\text { caso-controle ou de coorte }\end{array}$ \\
\hline $\begin{array}{l}\text { Revisão sistemática de estudos } \\
\text { qualitativos ou descritivos }\end{array}$ & NV & $\begin{array}{l}\text { Evidência proveniente de uma revisão sistemática de } \\
\text { estudos qualitativos ou descritivos }\end{array}$ \\
\hline Estudo descritivo ou qualitativo & NVI & Evidência de um único estudo descritivo ou qualitativo \\
\hline Opinião ou consenso & NVII & $\begin{array}{l}\text { Evidência proveniente da opinião de autoridades e } \\
\text { relatórios de comissões de especialistas /peritos }\end{array}$ \\
\hline
\end{tabular}

Fonte: Stillwell, Fineout-Overholt, Melnyk, Williamson, 2010, p.43.

Para melhor compreensão, as principais informações dos artigos que compuseram a revisão estão dispostas no Quadro 2.

Quadro 2 - Publicações relacionadas à temática Atendimento Pré-hospitalar à crise em saúde mental (n=7), segundo título, autores, objetivos, ano e nível de evidência, setembro de 2010 a setembro de 2020.

\begin{tabular}{|c|c|c|c|c|}
\hline Título & Autores & Objetivos & Ano & $\begin{array}{c}\text { Nível de } \\
\text { evidência }\end{array}$ \\
\hline $\begin{array}{l}\text { 1. Concepções de } \\
\text { profissionais de saúde do } \\
\text { serviço de atendimento } \\
\text { móvel quanto à urgência } \\
\text { psiquiátrica. }\end{array}$ & $\begin{array}{l}\text { Diego Bonfada, Jacileide } \\
\text { Guimarães, Andiara Araújo } \\
\text { Cunegundes de Brito }\end{array}$ & $\begin{array}{l}\text { Analisar as concepções dos profissionais } \\
\text { de saúde do Serviço de Atendimento } \\
\text { Móvel de Urgência de Natal-RN, Brasil, } \\
\text { sobre o atendimento às urgências } \\
\text { psiquiátricas }\end{array}$ & 2012 & N VI \\
\hline $\begin{array}{l}\text { 2. Atendimento pré- } \\
\text { hospitalar ás urgências } \\
\text { psiquiátricas por uso de } \\
\text { substancias psicoativas. }\end{array}$ & $\begin{array}{l}\text { Lívia Karla Sales } \begin{array}{r}\text { Dias, } \\
\text { Michelle }\end{array} \text { Alves } \\
\text { Vasconcelos, Ana Karina } \\
\text { Barbosa } \\
\text { Késia Marques Moncelos, }\end{array}$ & $\begin{array}{l}\text { Analisar a percepção de uma equipe } \\
\text { multidisciplinar quanto aos atendimentos } \\
\text { de urgências psiquiátricas associadas ao } \\
\text { uso de substâncias psicoativas realizados } \\
\text { pelo SAMU } 192 \text { em Sobral- Ceará. }\end{array}$ & 2013 & N VI \\
\hline $\begin{array}{l}\text { 3. Intervenção nas } \\
\text { situações de crise psíquica: } \\
\text { dificuldades e sugestões de } \\
\text { uma equipe de atenção pré- } \\
\text { hospitalar. }\end{array}$ & $\begin{array}{l}\text { Alexsandro } r \text { Barreto } \\
\text { Almeida, Eliane Regina } \\
\text { Pereira do Nascimento, } \\
\text { Jeferson } \\
\text { Gabriela Schweitzer. }\end{array}$ & $\begin{array}{l}\text { Conhecer como os profissionais de } \\
\text { atenção pré-hospitalar percebem as } \\
\text { intervenções nas pessoas em crise } \\
\text { psíquica. }\end{array}$ & 2014 & N VI \\
\hline
\end{tabular}




\begin{tabular}{|c|c|c|c|c|}
\hline $\begin{array}{l}\text { 4. Onde a reforma ainda } \\
\text { não chegou: ecos da } \\
\text { assistência às urgências } \\
\text { psiquiátricas }\end{array}$ & $\begin{array}{l}\text { Andiara } \\
\text { Cunegundes de } \begin{array}{r}\text { Araújo } \\
\text { Brito, }\end{array} \\
\text { Diego Bonfada, Jacileide } \\
\text { Guimarães }\end{array}$ & $\begin{array}{l}\text { Discutir o atendimento prestado às crises } \\
\text { psiquiátricas pelos profissionais de saúde } \\
\text { do Serviço de Atendimento Móvel de } \\
\text { Urgência (Samu) }\end{array}$ & 2015 & N VI \\
\hline $\begin{array}{l}\text { 5. Atendimento móvel de } \\
\text { urgência na crise psíquica e } \\
\text { o paradigma psicossocial }\end{array}$ & $\begin{array}{l}\text { Alexsandro } r \text { Barreto } \\
\text { Almeida, Eliane Regina } \\
\text { Pereira do Nascimento, } \\
\text { Jeferson Rodrigues, Maria } \\
\text { Terezinha Zeferino, Ana } \\
\text { Izabel Jatobá de Souza, } \\
\text { Patrícia Madalena Vieira } \\
\text { Hermida }\end{array}$ & $\begin{array}{l}\text { Conhecer a percepção e as intervenções } \\
\text { de equipes de suporte básico do serviço } \\
\text { de atendimento móvel de urgência na } \\
\text { situação da crise psíquica. }\end{array}$ & 2015 & N VI \\
\hline $\begin{array}{l}\text { 6. Atendimento pré- } \\
\text { hospitalar realizado pela } \\
\text { unidade de suporte básico } \\
\text { do SAMU a pacientes } \\
\text { psiquiátricos em } \\
\text { município da região } \\
\text { Catarinense. }\end{array}$ & $\begin{array}{l}\text { Luana Mara Rodrigues, } \\
\text { Marilene Nonnemacher } \\
\text { Luchtemberg, } \quad \text { Silvia } \\
\text { Salvador do Prado }\end{array}$ & $\begin{array}{l}\text { Identificar como os profissionais do } \\
\text { SAMU prestam atendimento a pacientes } \\
\text { psiquiátricos em um município da região } \\
\text { sul de Santa Catarina. }\end{array}$ & 2017 & N VI \\
\hline $\begin{array}{l}\text { 7. Concepções dos } \\
\text { enfermeiros frente à } \\
\text { utilização de protocolos de } \\
\text { urgência psiquiátrica no } \\
\text { atendimento pré-hospitalar } \\
\text { móvel }\end{array}$ & $\begin{array}{l}\text { Sisney Darcy Vaz da Silva, } \\
\text { Adriane Maria Netto de } \\
\text { Oliveira, Silvana Possani } \\
\text { Medeiros, Rúbia Gabriela } \\
\text { Fernandes } \\
\text { Luciano Garcia Lourenção, }\end{array}$ & $\begin{array}{l}\text { Conhecer as concepções do enfermeiro } \\
\text { frente à utilização de protocolos de } \\
\text { urgência psiquiátrica no atendimento } \\
\text { pré-hospitalar móvel. }\end{array}$ & 2020 & N VI \\
\hline
\end{tabular}

Fonte: Autores, a partir dos dados (2020).

Os sete artigos selecionados são de periódicos brasileiros. No que se refere ao período de publicação, os anos estão entre 2012 a 2020, destacando-se o ano de 2015 com duas publicações na temática pesquisada. Ressalta-se que quatro artigos (57.14\%) foram publicados em revistas da área de enfermagem e três (42.85\%) em revistas da área de saúde coletiva.

Quando classificados por níveis de evidências seguindo as recomendações de Stillwell et al. (2010) todos são de nível VI, sinalizando que o maior nível de evidência referiu-se aos estudos descritivos (não-ex-perimentais) ou com abordagem qualitativa.

É importante relatar que fazem parte das equipes de Atendimento Pré-hospitalar móvel, na unidade de suporte básico: técnicos em enfermagem e condutores, na unidade avançada: enfermeiro, médico e condutor.

Em resposta à pergunta norteadora verificou-se que uma das maiores dificuldades encontradas no atendimento em crise psíquica foi a falta de treinamento e conhecimento da equipe para desenvolver o serviço. Os profissionais sentem que não estão totalmente capacitados e instrumentalizados para realizar o atendimento em urgência psiquiátrica e ainda existe relato de insegurança, medo e de lacunas no conhecimento técnico e científico, uma vez que as ações estão embasadas em conhecimento empírico e pessoal sustentado no modelo biomédico (Silva et al., 2020; Almeida et al., 2014). A ideia do serviço de atendimento pré-hospitalar como de intervenção rápida e pontual também dificulta a apropriação das equipes para uma ação ampliada (Almeida, 2015).

Observa-se também o relato de que na maioria das ocorrências deste tipo há envolvimento do uso de substâncias psicoativas e por esse motivo, muitas vezes, há o uso exagerado de forças policiais devido à falta de preparo da equipe e do medo demonstrado (Dias et al, 2013; Bonfada et al., 2012; Brito et al., 2015).

A escassez de um programa de saúde mental eficiente, inexistência de leitos adequados ao atendimento e ausência de um hospital psiquiátrico que sirva de porta de entrada, são fatores citados que dificultam um bom atendimento pré-hospitalar 
em crise psíquica (Vasconcelos et al., 2013).

Em relação a organização para o atendimento, há o relato da necessidade da organização sistemática do trabalho baseado em uma rede de atenção e a falta de processos para o atendimento, já que os resultados apontaram que para atender o sujeito em crise psíquica é necessário todo esse suporte (Almeida, 2014).

Somente um artigo relata que os profissionais entrevistados estão preparados e capacitados para o atendimento em crise psíquica, mas ao mesmo tempo, uma parte deles afirma ter dificuldades na abordagem devido a agressividade do paciente (Rodrigues et al., 2017).

\section{Discussão}

O serviço de atendimento móvel de urgência (SAMU) foi normatizado e regulamentado no Brasil a partir de 2004 pelo decreto de $\mathrm{N}^{\circ} 5.055$, de 27 de abril, garantindo o atendimento precoce em situações de urgência e emergência em ambiente pré-hospitalar (Brasil, 2004).

A Portaria 2048/GM (Brasil, 2002) que regulamenta o atendimento das urgências e emergências, classifica a crise psíquica como situação de urgência, sendo atribuída a responsabilidade do atendimento ao SAMU, com suporte da polícia e bombeiros militares. A capacitação deve ser realizada no momento da implantação de todo serviço a um município, com no mínimo quatro horas de treinamento para o socorrista, ensino exclusivamente teórico, e duas horas teóricas e quatro horas práticas para o técnico de enfermagem (Brasil, 2002).

Para Fernandes et al. (2016) a crise se refere ao momento em que o indivíduo perde a capacidade de resolver determinado problema ou situação, ocorrendo um estado emocional de desequilíbrio, não conseguindo lidar com seus sentimentos evidenciados por perda, ameaças ou fatores psicológicos.

Quando trata-se do atendimento em crise no ambiente pré-hospitalar, a deficiência na formação e preparação do profissional pode acarretar problemas na qualidade do serviço prestado, evidenciando ações pautadas no modelo biomédico. Observa-se que a crise psíquica é uma situação de alta complexidade de difícil compreensão, que envolve o indivíduo, equipe de saúde e familiares e, todos estes fatores podem vir a influenciar na abordagem da crise, deixando claro que são necessários treinamentos mais amplos do que aquele previsto em lei e qualificação efetiva para o profissional.

Mesmo com a Política Nacional de Saúde Mental, que está pautada na mudança paradigmática de interpretar/responder o fenômeno da loucura, pode-se verificar um investimento na implantação de estruturas de Atenção Préhospitalar ao sujeito em crise psíquica, mas ao mesmo tempo, percebe-se uma fragilidade por parte de alguns gestores no processo de capacitação profissional para atender essa demanda e da estruturação da rede, especialmente ao que se refere a uma instituição específica de internação psiquiátrica. Um dos indicativos dessa dificuldade está relacionado principalmente à escassa literatura referente à atenção à crise.

Com a implementação da Reforma Psiquiátrica, foi instituída a Rede de Atenção Psicossocial (RAPS), articulando diferentes serviços, Centros de Atenção Psicossocial (CAPS), leitos de saúde mental em Hospital Geral, Serviços Residenciais Terapêuticos (SRTs), Unidades de Acolhimento (UA), atendimento em serviços de atenção básica, porém a falta de vagas para os atendimentos, a escassez de profissionais capacitados e insuficiência de medicamentos e leitos psiquiátricos dificultam o acolhimento do paciente (Barros; Tung; Maria, 2010).

$\mathrm{O}$ atendimento em saúde diante de uma crise, deve ser trabalhado de forma humana e integral, necessitando de profissionais qualificados que entendam as necessidades do indivíduo como primeira importância (Bonfada e Guimarães, 2012).

As emergências psiquiátricas são de caráter complexo e conflituoso, exigindo assim, equipe treinada para intervenção imediata que evite futuros agravantes ao indivíduo e aos que estão a sua volta (Deu-Bem et al., 2017). Ao profissional da saúde 
que atua nessa área são exigidas habilidades específicas, resolutivas e imediatas, tornando-se imprescindível especializar-se na temática a fim de agir de maneira adequada e criando o plano de ação para este momento de complexidade a fim de evitar que $\mathrm{o}$ atendimento se torne patologizante e estigmatizante.

A sensação de insegurança ou falta de conhecimento faz com que os profissionais de saúde utilizem mais as contenções medicamentosas e mecânicas, de forma indiscriminada durante a realização do manejo (Willrich et al., 2014). O uso de contenções deve ser tratado com cautela e enfatizado que esta não é a única alternativa para este tipo de atendimento. Considera-se esta prática um ato de preconceito, uma visão estigmatizada do paciente psiquiátrico e grande despreparo por parte da equipe. Há cada vez mais estudos mostrando que intervenções não coercitivas realizadas com genuíno comprometimento têm mais sucesso do que se imaginava inicialmente (Richmond et al., 2012).

Além da equipe de saúde, em situações de atendimento às urgências relacionadas às causas externas ou de pacientes em locais de difícil acesso, a portaria 2048/GM (Brasil, 2002) autoriza o uso de força policial no atendimento, caso o profissional da saúde ache necessário. Porém, segundo Jardim e Dimenstein (2008), o uso excessivo da polícia no atendimento às crises psíquicas ocorre devido ao estigma da agressividade do paciente psiquiátrico, fazendo com que o profissional menospreze o ideal e os valores defendidos na reforma psiquiátrica brasileira.

A abordagem e o manejo de qualidade ao paciente em crise são de suma importância para se ter uma boa resolução do caso. Um bom manejo, de acordo com os protocolos de Manejo de Crise em Saúde Mental do SAMU (Brasil, 2016), é aquele capaz de acolher tanto o paciente quanto a família, ter uma escuta aberta e qualificada, estabelecendo uma relação de confiança, estimulando a tranquilização do cenário, a fim de ter uma resolutividade de qualidade, levando a um consenso em relação à situação.

\section{Considerações Finais}

Conclui-se que os profissionais do Serviço de Atendimento Pré-Hospitalar ainda estão vinculados a um atendimento hospitalocêntrico, biomédico, de modelo manicomial, predominando o medo e uma lacuna no conhecimento técnico e científico. Apesar das mudanças adquiridas a partir da reforma psiquiátrica, o atendimento continua embasado na doença e no estigma que esta sugere.

Em síntese, a necessidade requer intervenções não somente no âmbito do trabalho e sim durante a formação nas instituições de saúde, fazendo-se necessário uma busca de um modelo mais próximo do paradigma psicossocial, desconstruindo o estigma do medo da loucura, que foi construído historicamente pela psiquiatria clássica.

Os estudos brasileiros relativos a este tema são restritos, o que pode indicar desinteresse por esta área do conhecimento e despreocupação dos pesquisadores em conhecer as reais necessidades dos profissionais que atuam no APH.

\section{Referências}

Almeida, A. B., Nascimento, E. R. P. do, Rodrigues, J., Zeferino, M. T., Souza, A. I. J. de, \& Hermida, P. M. V. Atendimento móvel de urgência na crise psíquica e o paradigma psicossocial. Texto \& Contexto - Enfermagem, 24(4), 1035-1043.

Barbosa, D. J., Gomes, M. P., Souza, F. B. A. de, \& Gomes, A. M. T. Fatores de estresse nos profissionais de enfermagem no combate à pandemia da COVID19: síntese de evidências. Rev. Ciências Saúde, 118(98), 178-190.

Barros, R. E. M., Tung, TengChei, \& Mari, J. de J. Serviços de emergência psiquiátrica e suas relações com a rede de saúde mental brasileira. Rev. Bras. Psiquiatr, 32 .

Bonfada, D. Serviço de Atendimento Móvel de Urgência (SAMU) e a assistência às urgências psiquiátricas. 2010.148 f. Dissertação (Mestrado) - Curso de Programa de Pós-Graduação em Enfermagem, Universidade Federal do Rio Grande do Norte, Natal, 2010.

Bonfada, D., \& Guimarães, J., \& Brito, A. A. C. de. Concepções de profissionais de saúde do serviço de atendimento móvel quanto à urgência psiquiátrica. Revista da Rede de Enfermagem do Nordeste, 13(2), 309-320.

Bonfada, D., \& Guimarães, J. Serviço de atendimento móvel de urgência e as urgências psiquiátricas. Psicologia em estudo, 17(2), 227-236. 
Research, Society and Development, v. 10, n. 11, e275101119423, 2021

(CC BY 4.0) | ISSN 2525-3409 | DOI: http://dx.doi.org/10.33448/rsd-v10i11.19423

Brasil. Ministério da Saúde. Portaria no 2048/GM de 05 de novembro de 2002. Dispõe sobre o funcionamento dos serviços de urgência e emergência. Diário Oficial da União, p. 50, 12 dez. 2002.

Brasil. Ministério da Saúde. Decreto n ${ }^{\circ} 5.055$ de 27 de abril de 2004. Institui o serviço de atendimento móvel de urgência - SAMU, em municípios e regiões do território nacional, e dá outras providências. Diário Oficial da União, Brasília, DF, 28 mar. 2004.

Brasil. Ministério da Saúde. Reforma Psiquiátrica e política de saúde mental no Brasil. Ministério da Saúde, 2005.

Brasil. Ministério da Saúde. Secretaria de Atenção à Saúde. Protocolos de Intervenção para o Samu 192 - Suporte Básico de Vida.: Ministério da Saúde, 2015.

Brito, A. A. C., Bonfada, D., \& Guimarães, J. Onde a reforma ainda não chegou: ecos da assistência às urgências psiquiátricas. Physis: Revista de Saúde Coletiva, 25(4), 1293-1312.

Del-Ben, C. M., Sponholz-Junior, A., Mantovani, C., Faleiros, M. C. de M., Oliveira, G. E. C., Guapo, V. G., \& Marques, J. M. de A. Emergências psiquiátricas: manejo da agitação psicomotora e avaliação de risco suicida. Medicina (Ribeirão Preto. Online), 50(1), 98-112.

Dias, L. K. S., Vasconcelos, M. A., Vasconcelos, A. K. B., \& Moraes, K. M. Atendimento pré-hospitalar às urgências psiquiátricas por uso de substâncias psicoativas. SANARE, 12. 47-53.

Fernandes, M. A., Pereira, R. M. F., Leal, M. S. M., Sales, J. M. F. de, \& Silva, J. S. e. Cuidados de enfermagem ao paciente psiquiátrico na urgência de um hospital geral. Revista de Enfermagem da UFPI, 5(2), 41-45.

Jardim, K., \& Dimenstein, M. A crise na rede do Samu no contexto da Reforma Psiquiátrica. Saúde em Debate, 32(78/79/80), 150-160.

Rodrigues, L. M., Luchtemberg, M. N., \& Prado, S. S. do. Atendimento pré-hospitalar realizado pela Unidade de Suporte Básico do SAMU a pacientes psiquiátricos em um município da região sul catarinense. Revista Inova Saúde, 6(2), 1-3.

Richmond, J. S., Berlin, J. S., Fishkind, A. B, Holloman, G. H., Zeller, S. L., Wilson, M. P., Rifai, M. A., \& Ng, A. T. Verbal de-escalation of the agitated patient: consensus statement of the American Association for Emergency Psychiatry Project BETA de-escalation workgroup. The Western Journal of Emergency Medicine, 13(1), 17-25.

Silva, S. D. V. da, Oliveira, A. M. N. de, Medeiros, S. P., Salgado, R. G. F., Lourenção, \& L. G. Concepções dos enfermeiros frente à utilização de protocolos de urgência psiquiátrica no atendimento pré-hospitalar móvel. Revista Enfermagem Uerj, 28, e 50191.

Stillwell, S., Fineout-Overholt, E., Melnyk, B. M., \& Williamson, K. Fineout-Overholt. Searching for the evidence: strategies to help you conduct a successful search. American Journal of Nursing, 110(5), 41, 2010.

Willrich, J. Q., Kantorski, L. P., Antonacci, M. H., Cortes, J. M., \& Chiavagatti, F. G. Da violência ao vínculo: construindo novos sentidos para a atenção à crise. Revista Brasileira de Enfermagem, 67(1), 97-103. 